\title{
Design of Wireless Sensor Network Node for Monitoring Rice Field
}

\author{
Hua-Qiang Chen, Jiang-Peng Weng, Feng-Lian Tie \\ College of Electronic Engineering, South China \\ Agricultural University, China \\ E-mail: chenhuaqiang@163.com, \\ vmishiwjpv@163.com, 65517386qq.com
}

\author{
Bao-Xia Sun \\ Guangdong Engineering Polytechnic, China \\ E-mail: sunbaoxia@126.com
}

\author{
Wei-Xing Wang \\ College of Electronic Engineering, South China Agricultural University, China \\ Guangdong Engineering Research Center for Monitoring Agricultural Information, China \\ Key Laboratory of Information Acquisition and Application in Agriculture, Guangzhou Science Technology and Innovation \\ Commission, China \\ E-mail: weixing@scau.edu.cn
}

\begin{abstract}
A wireless sensor network (WSN) node is designed for monitoring rice field in order to solve the existing problems of poor real-time performance, Low system stability and large monitoring area, etc. The node uses a STM32F103VET6 as a processing core, and a WLK01L39 RF chip is used in wireless communication module, while the sensor module is composed of the air temperature and humidity sensor, Light intensity sensor and soil moisture sensor. uC/OS-II is applied as operation system to realize multitask scheduling running. The sensor node applies a mechanism as sleeping and waking up work modes to reduce power consumption. The mean current consumption of sensor nodes raged from $23.24 \mathrm{~mA}$ to $32.82 \mathrm{~mA}$ under operating mode and $0.026 \mathrm{~mA}$ under sleeping mode. The results of a two-week networking experiment indicated that the average PLR (Packet Loss Rate) of network was $0.76 \%$. In conclusion, the design of sensor node is suitable for effective monitoring of rice field.
\end{abstract}

Keywords-wireless sensor network; rice field; information acquisition; low power consumption; node design

\section{INTRODUCTION}

In our country, the planting area and production of rice occupy the first position among main grain crops $[1,2,3]$. However, some problems have been restricting the development of rice industry, such as the backward production technology and small production scale. Wireless sensor network (WSN), which is considered as one of the most important technologies in the twenty-first Century, is a new type of information acquisition and processing technology which can be applied in the agricultural field [4]. WSN is generally composed of a large number of sensor nodes with specific functions. Because of its simple deployment, intensive layout, low cost, no need for on-site maintenance and so on, it provides convenience for the data acquisition of environmental science research. The combination of WSN and farmland environment parameter sensor [4] can realize the real-time online monitoring of large areas of farmland environment parameter, which is significant in accelerating the agricultural production information degree of our country to predict rice drought, plant diseases and insect pests. There have been many successful application examples at home and abroad[5,6,7,8,9].

There are many kinds of WSN nodes, and it is necessary to design and develop different nodes basing on the characteristics in different monitoring environment. Huang Jianqing[6]et al. develop a collection node and gateway node for the problem of poor flexibility and wiring difficulty for the water quality automatic detection system They construct a remote on-line monitoring system for aquaculture and realize the remote on-line monitoring of dissolved oxygen, $\mathrm{pH}$ value and temperature parameters in the water body. Zhang Jing[9] et al use CC2530 chip and peripheral interface to construct the hardware and the Z-Stack protocol stack to compile the system bottom software. They developing the software of the greenhouse environment monitoring system based on the VB software platform, and analyzed the transmission characteristics of CC2530 chip. However, the network size and connectivity may be restricted for the node communication distance which is only $60 \mathrm{~m}$. Li Xiaomin[10] et al. design a wireless sensor node with adaptive transmit power according to the changes of environmental factors in rice growing process and the energy consumption of sensor nodes in wireless radio frequency stage, but the node design has the specificity of the monitoring environment. WSN nodes need to be low-power because they are generally powered with batteries. Therefore, the subject of this paper is how to use fewer nodes to achieve efficient and reliable field information collection [11].

This paper designs a WSN node, whose monitoring 
objectives are air temperature and humidity, light intensity and the soil water temperature, according to the characteristics of wide monitoring environment, abundant solar energy and few building barriers. In order to meet the application requirements of real-time on-line monitoring in rice field environment, the node is designed to realize the long distance and effective communication, and the energy self-supply continuously and stably.

\section{HARDWARE DESIGN OF WSN NODES}

\section{A. Hardware Structure of Sensor Node}

Nodes constitute a complete wireless sensor network in form of self-organization. They send the environmental monitoring information of rice field through single hop or multi hop transmission to the monitoring center via the gateway node. In this way, it can achieve the monitoring environment parameters acquisition of rice field [12]. The acquisition node consists of a processor module, a power supply, a solar charging module, a sensor module and a wireless radio frequency module. The WSN node structure is shown in Fig. 1. The physical map of the sensor node is shown in Fig. 2.

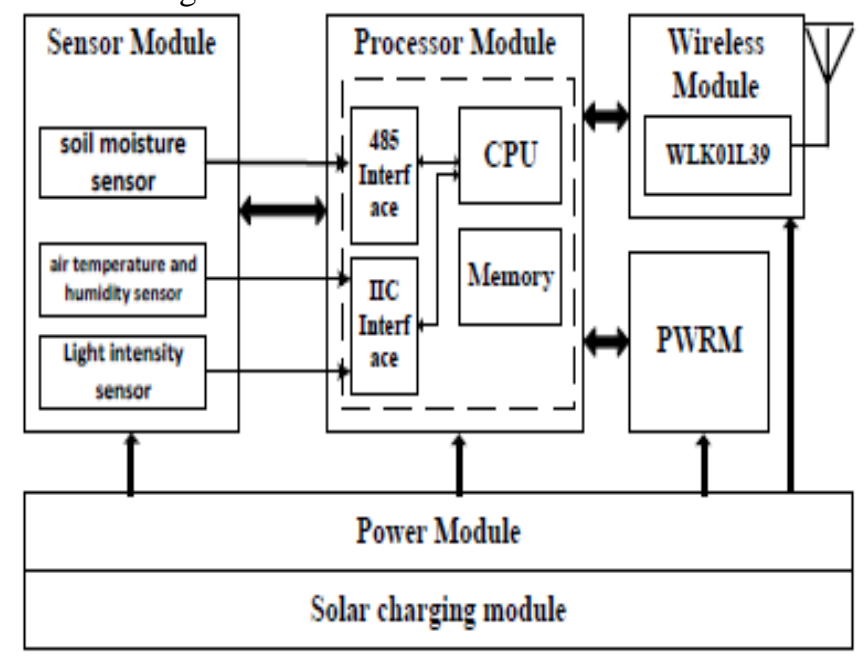

Figure 1. The structural of WSN node.

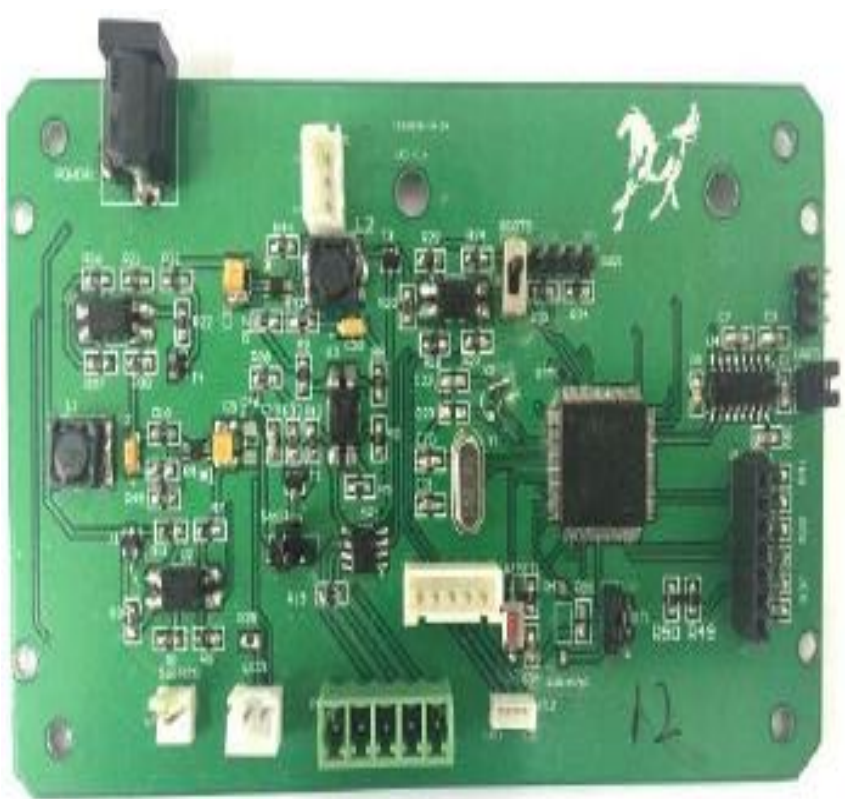

Figure 2. Sensor node.

\section{B. Processor Module}

In order to meet the design requirements of superior performance and low power consumption, the microprocessor uses STM32F103VET6. It has high command execution efficiency and strong anti-jamming capability. What's more, it supports standby sleep and has the functions of equipment management, task scheduling and data fusion processing, etc.

\section{Power Supply and Solar Charging Module}

Since nodes are put in the rice field without electricity supply and it takes a long time to work, the design of the power supply module is crucial to the whole system. Considering the node's endurance, the design uses rechargeable lithium battery $(12 \mathrm{~V}, 10000 \mathrm{MAH})$. In view of the input voltage of the system and the maximum current at work, TPS62170 is used as the voltage conversion chip, which can generate stable $5 \mathrm{~V}$ voltage to supply power for the sensor and stable $3.3 \mathrm{~V}$ voltage for the processor, radio frequency module and other equipment by selecting the appropriate resistor between the FB pin and the VOS pin of the chip. At the same time, taking into account the fact that rice field is usually sunny, this system use solar charging panels and solar charge controller for battery charging to prolong the lifetime of nodes.

\section{Sensor Module}

The sensor module is mainly responsible for the collection of air temperature and humidity, light intensity, soil temperature and humidity in the rice field. According to the accuracy of the data parameters and the field application needs, the design uses HTU21D temperature and humidity sensors produced by French Humirel Company, light intensity sensor BH1750FVI, as well as soil moisture temperature sensor SMTS-II-485. 


\section{E. Radio Frequency Module}

The wireless communication module is the bridge of data transmission among nodes. Due to wide monitoring area, WLK01L39, which is with low power consumption, strong anti-interference ability and far transmission distance, is used as a radio frequency chip. In addition, its stable transmission distance is up to $2 \mathrm{~km}$ in the open environment.

\section{SOFTWARE DESIGN OF WSN NODES}

The performance of the wireless sensor network nodes and the stability of the network are directly affected by the software design. Monitoring in rice field is characterized by long cycle, strong disturbances and small quantity of data in single transmission. Considering the characteristics, a software system based on the uC/OS-II is designed according to the hardware platform above mentioned. Due to the use of embedded operating system, the design greatly reduces the workload of software engineering in complex application system and improves the quality of software. In embedded applications, CPU must work reliably. Even if the system goes wrong for some reason, it should be restored automatically. The watchdog is used to reset the system if it goes wrong.

\section{A. Design of Communication Protocol between Nodes}

Limited by the node energy, network computing and processing capacity and other resources, design of WSN communication protocol is particularly important $[13,14]$. The design reduces the cost of energy and data transmission delay by means of routing algorithm, time synchronization algorithm and standby wake-up mechanism, and thus the allocation of WSN resources can be optimized.

\section{1) Routing algorithm design:}

The routing protocol designed in this paper is based on the LEACH protocol. This system is aimed at the large rice field; therefore the design uses the clustering routing algorithm. Cluster head is randomly selected in each round. Data packets of nodes in a cluster are sent to the cluster head and data packets between clusters can be forwarded to the gateway node through multi hop of cluster heads. Cluster head nodes first check their own record after receiving a new packet. If the packet has been forwarded, they will not be forwarded again. In this way, the waste of energy caused by forwarding the same data packets can be avoided. The structure of packets sent to the cluster head by sensor nodes and packets sent to the gateway node by cluster head is shown in Fig. 3.

\begin{tabular}{|c|c|c|c|c|c|c|c|c|}
\hline Data & Cluster & Node & Sensor & Sensor & Sensor & Battery & Jump & CRC \\
Flag & Num & Num & 1 & 2 & 3 & Voltage & Num & (a) \\
\hline
\end{tabular}

Figure 3. Sensor node packet structure.

\section{2) Time synchronization algorithm design:}

After the gateway node obtain the current time and the standby time from the remote server, it will process the information packet of the synchronous time, and then send to the cluster heads and nodes in clusters through broadcast. Each node in clusters receives the synchronous packet, which will be parsed into the current time and the alarm clock time, then set the RTC clock register before entering the low power standby mode. The structure of synchronization time information packet is shown in Fig. 4.

\begin{tabular}{|l|c|c|c|}
\hline $\begin{array}{c}\text { Syn } \\
\text { Flag }\end{array}$ & $\begin{array}{c}\text { Current } \\
\text { Time }\end{array}$ & $\begin{array}{c}\text { Alarm } \\
\text { Time }\end{array}$ & CRC \\
\hline
\end{tabular}

Figure 4. Synchronization time packet structure.

\section{3) Standby wake-up mechanism:}

In the network, each node has its local clock. After the system starts, the network will provide a common time stamp for local clocks of all nodes and it will be on standby or be awakened at the right time. By means of this standby mechanism, the whole network can save the cost and reduce the energy consumption.

\section{B. Application Design}

The version of small embedded real-time system in acquisition nodes is V2.91 uC/OS-II. The system is responsible for preemptive real-time multi task scheduling. Tasks communicate with each other through a signal. The whole system is divided into several parts, including initialization task, acquisition task, receiving and forwarding packets (including synchronization packet and data packet) task, sending data package task, watching dog task and standby task. The system block diagram of application is shown in Fig. 5.

Here is the process of the system application in per round. Firstly, the initialization task is executed to initialize the hardware resources. Secondly, acquisition signal is sent to acquisition task and collecting starts. Thirdly, after the sending data package task receives the sending signal, the collected data is packaged and sent to the cluster head node through the wireless module. When the receiving and forwarding package task receives packages from other nodes, it will determine whether forward the packet or not. If the received packet is synchronizing information, a standby signal will be sent to standby task. Then standby task is executed to configure the Real-Time Clock (RTC) register. Finally, the system will enter the sleep mode, waiting for the next RTC alarm clock to wake up and start a new round of work.

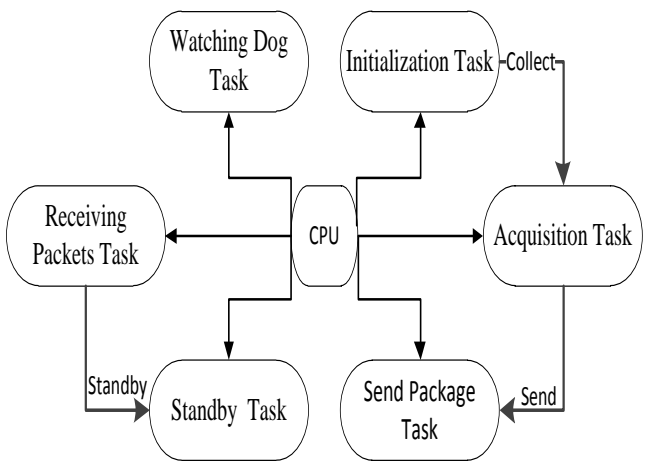

Figure 5. The application system diagram. 


\section{TEST AND RESULT ANALYSIS}

\section{A. Node Hardware Performance Test}

Node hardware performance test mainly refers to low power performance test. We measured the working current in the system in different working modes using a high precision ammeter accessed to node circuit. After repeatedly testing, sensor nodes record data and find the average value. The test results are shown in Table 1. Results show that the sleep \& wake-up mechanism effectively extending the life cycle of nodes and achieve the requirements of low power consumption.

TABLE I. POWER TEST RESULTS OF SENSOR NODE

\begin{tabular}{ccccc}
\hline Test node & Standby & $\begin{array}{c}\text { Data } \\
\text { Collect }\end{array}$ & $\begin{array}{c}\text { Data } \\
\text { Send }\end{array}$ & $\begin{array}{c}\text { Data } \\
\text { Receive }\end{array}$ \\
\hline Collectors $/ \mathrm{mA}$ & 0.026 & 32.82 & 27.35 & 23.24 \\
\hline
\end{tabular}

\section{B. Network Packet Loss Rate Test}

In this networking test, 10 sensor nodes are divided into two clusters and a gateway node. The sensor nodes collect and send data once every $10 \mathrm{~min}$, and then enter the receiving mode after the data packet is sent. Finally, they will enter the sleep state after receiving the synchronization information from the gateway node. The test data of $14 \mathrm{~d}$ are statistically analyzed and the results are shown in table 2 . The results show that the system is stable and reliable, and the average packet loss rate of the network is $0.76 \%$

TABLE II. PLR VALUES OF NETWORK

\begin{tabular}{ccccc}
\hline $\begin{array}{c}\text { Cluster } \\
\text { Num. }\end{array}$ & $\begin{array}{c}\text { Node } \\
\text { Num. }\end{array}$ & $\begin{array}{c}\text { Send } \\
\text { Packet } \\
\text { Num. }\end{array}$ & $\begin{array}{c}\text { Receive } \\
\text { Packet } \\
\text { Num. }\end{array}$ & $\begin{array}{c}\text { Packet } \\
\text { Loss } \\
\text { Rate /\% }\end{array}$ \\
\hline 1 & 01 & 2016 & 2005 & 0.54 \\
1 & 02 & 2016 & 2001 & 0.74 \\
1 & 03 & 2016 & 1999 & 0.84 \\
1 & 04 & 2016 & 1995 & 1.04 \\
1 & 05 & 2016 & 2004 & 0.59 \\
2 & 01 & 2016 & 2002 & 0.69 \\
2 & 02 & 2016 & 1997 & 0.94 \\
2 & 03 & 2016 & 2001 & 0.74 \\
2 & 04 & 2016 & 1995 & 1.04 \\
2 & 05 & 2016 & 2006 & 0.49 \\
\hline
\end{tabular}

\section{CONCLUSIONS}

To solve the problems, such as poor real-time and low system stability, of on-line monitoring on large rice fields, a kind of rich field's node based on wireless sensor network is designed and its related performance test is carried out. Test results show that:

By means of component selection, hardware circuit design and program design, a sensor node for the on-line monitoring on large rice field is developed. The node can realize the real-time collection and remote transmission of water quality data.

Sensor nodes have low power consumption in the monitoring process. The current of sensor nodes in the system sleep phase, data acquisition phase, data transmission phase and data reception phase is $0.026 \mathrm{~mA}$, $32.82 \mathrm{~mA}, 27.35 \mathrm{~mA}$ and $23.24 \mathrm{~mA}$ respectively. Most of the time the sensor nodes are in the standby sleep phase during the system running time, so the system can work stably in a long term.

Due to the close distance between nodes in this test, the average packet loss rate is $0.76 \%$. Network packet loss rate relates closely with node deployment location, antenna height and other factors. In practice, the network packet loss rate can be reduced by changing the transmission power of the wireless module, adjusting the height of the antenna, increasing the routing node and so on.

\section{ACKNOWLEDGEMENT}

This research was financially supported by Natural Science Foundation of Guangdong Province, China (2014A030313451); Science and Technology Planning Project of Guangdong Province, China (2015A020209013)

\section{REFERENCES}

[1] Huizhe Chen, Defeng Zhu, The Rice Production and Ecosystem in the World, HYBRID RICE. 05(2003) 4-7.

[2] Zhenhuan Liu, Zhengguo Li, Pengqin Tang, et al. Spatial-temporal changes of rice area and production in China during 1980-2010, Acta Geographica Sinica. 68(5)(2013)680-693.

[3] Hongcheng Zhang, Jinlong Gong, Research Status and Development Discussion on High-Yielding Agronnmy of Mechanized Planting Rice in China, Scientia Agricultura Sinica. 07 (2014) 1273-1289.

[4] Xiaojun Qiao, Xin Zhang, Cheng Wang, Application of the wireless sensor networks in agriculture, Transactions of the CSAE.S2 (2005)232-234.

[5] Antai Han, Yong He, Zhiqiang Chen, et al. Design of Distributed Precision Irrigation Control System Based on Wireless Sensor Network for Tea Plantation, Transactions of the Chinese Society for Agricultural Machinery. 09 (2011)173-180.

[6] Jianqing Huang, Weixing Wang, Sheng Jiang, et al. Development and test of aquacultural water quality monitoring

[7] system based on wireless sensor network, Transactions of the Chinese Society of Agricultural Engineering. 04(2013) 183-190.

[8] Jin $\mathrm{Hu}$, Hongpan Fan, Haihui Zhang, et al. Design of regulation system of light environment in greenhouse based on wireless sensor network, Transactions of the Chinese Society of Agricultural Engineering.04(2014) 160-167.

[9] Sheng Jiang, Weixing Wang, Daozong Sun, et al. Design of energy self-sufficient wireless sensor network node for orchard information acquisition, Transactions of the Chinese Society of Agricultural Engineering. 09(2012) 153-158.

[10] Jing Zhang, Qiliang Yang, Zhenyang Ge, et al. WSN monitoring system for greenhouse environmental parameters

[11] and CC2530 transmission characteristics, Transactions of the Chinese Society of Agricultural Engineering. 07(2013) 139-147.

[12] Xiaomin Li, Ying Zang, Xiwen Luo, et al. Design of WSN node with adaptive transmitting power for rice field, Transactions of the Chinese Society of Agricultural Engineering. 07(2014) 140-146. 
[13] Bo Zhang, Xiwen Luo, Yubin Lan, et al. Agricultural environment monitor system based on UAV and wireless sensor networks, Transactions of the Chinese Society of Agricultural Engineering. 2015(2015) 176-182.

[14] Baoxia Sun, Weixing Wang, Gang Lei, et al.Real-time Monitoring System for Paddy Environmental Information

[15] Based on Wireless Sensor Network, Transactions of the Chinese Society for Agricultural Machinery. 09(2014) 241-246.
[16] Changiiang Jiang, Weiren Shi, Xianlun Tang, et al. Energy-Balanced Unequal Clustering Routing Protocol for Wireless Sensor Networks, Journal of Software. 05(2012)1222-1232.

[17] Tieliu Liu, Yongqun Wu, Energy Optimized Approach Based on Clustering Routing Arithmetic For Wireless Senor Networks, CHINESE JOURNAL OF SENSORS AND ACTUATORS.05(2011) 764-770. 